\title{
reviscafuences
}

ISSN: 1575-7072 | e-ISSN: 2172-7775

Páginas: 88-104

Recibido: 2019-05-17

Revisado: 2019-08-28

Aceptado: 2019-11-04

Preprint: 2020-04-20

Publicación Final: 2020-06-15 www.revistascientificas.us.es/index.php/fuentes/index

DOI: https://doi.org/10.12795/revistafuentes.2020.v22.i1.08

\section{Validación y fiabilidad del Cuestionario sobre Acoso entre Estudiantes Universitarios (QAEU)}

\section{Validation and reliability of the Questionnaire on Harassment among University Students (QAEU)}

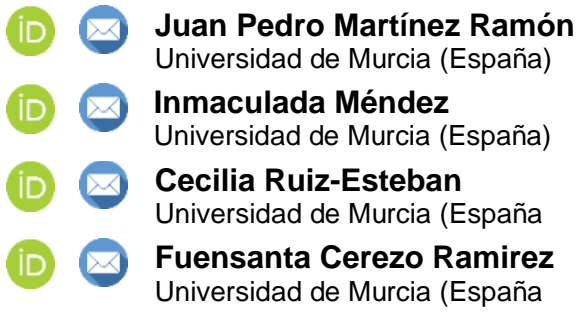

\section{Resumen}

El acoso entre iguales es una realidad en las enseñanzas superiores. Las nuevas tecnologías suponen un vehículo para la aparición y propagación del fenómeno. El objetivo de este estudio fue analizar la validez y fiabilidad del Cuestionario sobre Acoso entre Estudiantes Universitarios (QAEU), herramienta diseñada para su detección. Se trata de una prueba psicométrica compuesta por tres escalas: objeto, agente y observador. En el estudio participaron 765 estudiantes de Grado y Máster de diversas titulaciones de una universidad del sureste español con una media de edad de $23.27(D T=$ .81), siendo el $72.7 \%$ mujeres. Los resultados mostraron una fiabilidad Alfa de Cronbach de .970. Los análisis estructurales, para confirmar la existencia de constructos en el cuestionario (tipo de acoso, vías por las que se produce, momento), presentaron un modelo robusto, tanto para las escalas de víctima, agresor o como observador. En conclusión, el QAEU es un instrumento con alta consistencia interna y válido para su uso en investigación, situaciones prácticas y en ámbitos de innovación.

\begin{abstract}
Harassment among peers is a reality framed in higher education. In addition, new technologies can be a vehicle for the appearance and propagation of the phenomenon. The main objective in this research was to analyse the validity and reliability of the Questionnaire on Harassment among University Students (QAEU in Spanish), a tool designed for its detection. It is a psychometric tool composed by three scales: object, agent and bystander. There were 765 students of Degree and Master from a university of the Spanish southeast with an average age of $23.27(S D=.81)$, with $72.7 \%$ women. The results showed a reliability by Cronbach' Alpha of .970. The structural equations model, analyses for confirming the constructs of the questionnaire (forms of aggression, ways and moment of aggression), presented robust model for victim scale, aggressor scale and even for bystanders scale. In conclusion, the QAEU is a tool with a high internal consistency and is valid for being used in research, practical situations and innovation areas.
\end{abstract}

\section{Palabras clave / Keywords}

Acoso; ciberacoso; validación; fiabilidad; universitarios.

Bullying; cyberbullying; validation; reliability; university students. 


\section{Introducción}

Las universidades precisan de herramientas para detectar el acoso eficazmente y evitar los efectos perjudiciales de su cronicidad (Halpern, Piña y Vásquez, 2017; Kopecký, 2013; Alonso-Martín, 2009). No obstante, la información referente a las propiedades psicométricas de los instrumentos para medir la violencia entre iguales es escasa (López, 2016), a pesar de suponer una realidad en las aulas. En esta línea, Perry y Blincoe (2015) encuentran que el $63.35 \%$ de los estudiantes de niveles superiores ha sido testigo de situaciones de acoso y Kokkinos, Antoniadou y Markos (2014) informan que más de la mitad de los universitarios ha estado implicado o relacionado en ciberacoso. Recientemente se ha encontrado que el $18.6 \%$ ha sido cibervíctima y el $19.4 \%$ ciberagresor (Martínez-Monteagudo, Delgado, Inglés y GarcíaFernández, 2019).

La mayoría de instrumentos de evaluación se centran en etapas no universitarias (Avilés, 2009; Cerezo, Sánchez, Ruiz-Esteban y Arense, 2015; Garaigordobil, 2015; Gascón-Cánovas, Ruso, Cózar y Heredia, 2016; Gómez-Ortiz, Del Rey, Romera y Ortega-Ruiz, 2015; Gómez-Ortiz, Romera-Félix y Ortega-Ruiz, 2017; Herrera-López, Romera y Ortega-Ruiz, 2017; Magaz, Chorot, Santed, Valiente y Sandín, 2016; Vera, Vélez y García, 2017) siendo escasos los estudios en entornos universitarios (Hoyos, Llanos y Valero, 2012; López, 2017; Sinkkonen, Puhakka y Merilänen, 2014).

Debido a dicha escasez de recursos en la educación superior, una estrategia eficaz es la de adaptar instrumentos diseñados inicialmente para etapas anteriores (Trujillo y Romero-Acosta, 2016). Tal es el caso del "Cyberbullying. Screening de acoso entre iguales" de Garaigordobil (2013) el cual va dirigido inicialmente a estudiantes de 12 a 18 años y mide la presencia de acoso tradicional o presencial y tecnológico o ciberacoso, analizando las conductas sociales, psicológicas, verbales y físicas asociadas a cada una. Debido a que el cuestionario abarca hasta los 18 años, sería posible adaptarlo a población adulta, tal como realizan Hoyos, Romero, Valega y Molinares (2009) con el Informe Nacional del Defensor del Pueblo sobre Violencia Escolar (2000).

Diseñar un instrumento en el ámbito universitario que considere el acoso y el ciberacoso implica tener en consideración tanto las manifestaciones en las que estos pueden darse como algunas de las variables sociodemográficas relacionadas, incluyendo: a) tipos de maltrato (verbal, exclusión...) (Hoyos et al., 2009); b) las vías de comunicación, el uso de dispositivos o la creación de grupos (Ortega-Ruiz, Del Rey y Casas, 2016); c) los motivos y causas (Perry y Blincoe, 2015); d) el sexo (Romera, Cano, García-Fernández y OrtegaRuiz, 2016); e) la procedencia (Perry y Blincoe, 2015); f) la edad (Hoyos et al., 2009); y el rol (López, 2016; Martínez-Monteagudo et al., 2019).

Cabe destacar que los instrumentos que se diseñen deben detectar eficazmente tres agentes fundamentales: agresor, víctima y observador (Hoyos et al., 2009; Sinkkonen et al. 2014). Si bien es cierto que estos roles pueden configurarse en primaria se mantienen hasta la adultez (Bender y Lösel, 2011) llegando a entornos universitarios.

Por su parte, Sinkkonen et al. (2014) recogen 2850 encuestas de universitarios en las que otorgan a la observación un papel destacado en la estructura psicométrica de sus dimensiones. Por tanto, queda expuesta la utilidad de crear herramientas dirigidas a los estudios superiores, que consideren los tres tipos de actores en el diseño de las escalas.

De cara a la administración de instrumentos de detección de acoso y ciberacoso a día de hoy, ésta también puede realizarse a través de la modalidad online, la cual, puede favorecer la libertad de expresión en contraposición a la impresa (Ortega-Ruiz et al., 2016). Por ello, se considera útil dar la opción de realizarse por una u otra vía en aras de reducir el efecto de deseabilidad social. A modo de ilustración se expone el estudio de Faucher, Jackson y Cassidi (2014), quienes administran una encuesta online sobre las tecnologías de la información y la comunicación a 1733 estudiantes universitarios para investigar el ciberacoso.

Para dar respuesta a las necesidades provenientes de la educación superior se ha creado el Cuestionario sobre Acoso entre Estudiantes Universitarios (QAEU) considerando los tres principales actores del acoso y ciberacoso. EI QAEU utiliza un cuestionario autocumplimentado lo cual puede resultar más útil que la observación directa, las entrevistas o las dinámicas de grupo al fomentar la sinceridad en la respuesta y permitiendo recoger mucha información en un corto espacio de tiempo, a través de su administración tanto tradicional como online.

Dicho esto, el objetivo general de esta investigación ha sido validar un cuestionario diseñado para detectar la presencia de acoso y ciberacoso entre iguales en el ámbito universitario delimitando la figura de acosador, acosado y observador. Para ello, se proponen los siguientes objetivos específicos: (1) Analizar la consistencia interna de las escalas y (2) diseñar un modelo de ecuaciones estructurales validando la existencia de los tres 
constructos que configuran el QAEU (como objeto de las agresiones, como agente de las agresiones y como observador de las agresiones).

\section{Método}

\subsection{Instrumento}

El Cuestionario sobre Acoso entre Estudiantes Universitarios (QAEU) es un instrumento psicométrico elaborado partiendo de la adaptación de otros instrumentos. Por un lado, el Cuestionario para Estudiantes (Hoyos et al, 2009), el cual, considera nuevamente tres roles (víctima, agresor y testigo), tiene 19 ítems y está adaptado a la población universitaria. Dicho cuestionario obtiene una alta fiabilidad con un Alfa de Cronbach igual a .801 (Hoyos et al, 2012). Parte originalmente del Informe Nacional del Defensor del Pueblo sobre Violencia Escolar (2000) realizado por la Universidad de Madrid. Es seleccionado por su fiabilidad y por partir a su vez de un cuestionario pilotado con población española.

Por otro lado, se ha utilizado el instrumento "Cyberbullying. Screening de acoso entre iguales" de Garaigordobil (2013) el cual consta de 45 ítems que valoran 15 conductas de ciberacoso y cuatro índices (victimización, perpetración, observación y victimización agresiva). Posteriormente, a través del análisis factorial se halla la existencia de tres factores tanto para acoso (víctima, agresor y observador) como ciberacoso (cibervictimización, ciberagresión y ciberobservación). Este instrumento es seleccionado por su alta consistencia interna en la medición del acoso y ciberacoso. Asimismo, el hecho de que sus destinatarios sean estudiantes castellanoparlantes de 12 a 18 años facilita la adaptación del vocabulario a población adulta. EI QAEU consta de 135 ítems distribuidos en tres escalas junto con una serie de variables sociodemográficas (véase Anexo). Este último aspecto está compuesto por seis cuestiones (edad, sexo, facultad, tipo de estudios, curso y país de procedencia). La primera escala, "como objeto de las agresiones», consta de 49 ítems distribuidos entre acoso (tipo, quién, lugar, momento, motivo) y ciberacoso (tipo, vía o medio, momento, motivo, informador y apoyo). La segunda escala, «como agente de las agresiones», tiene 35 ítems distribuidos entre agresión directa o acoso (tipo, objeto o dirección, momento y causa) y ciberacoso (acto, vía, objeto y motivos). Finalmente, la tercera escala, "como observador/a de las agresiones», consta de 45 ítems repartidos entre observación de la agresión directa o acoso (tipo, objeto, momento y motivos) y ciberacoso (tipo, vía, excluidos, causas, informados y ayuda).

A excepción de las cuestiones sociodemográficas y de los ítems del QAEU 7, 29, 56, 73, 91 y 108 que son dicotómicos (sí o no), el resto se cumplimenta a través de una escala tipo Likert de cuatro opciones: (a) nunca, (b) a menudo, (c) algunas veces y (d) siempre. En cada una se distribuyen los ítems de forma que aproximadamente la primera mitad analiza la posibilidad de acoso y la otra el ciberacoso. Su administración puede ser individual o colectiva y va destinado a alumnado universitario. Ejemplo de ítem dicotómico utilizado: "En alguna ocasión he sufrido agresión directa". Ejemplo de ítem de frecuencia: "Las agresiones se producen por cuestiones académicas".

\subsection{Diseño y procedimiento}

Investigación instrumental de acuerdo con la nomenclatura realizada por Montero y León (2007). Para el diseño del cuestionario, se realizó un análisis previo de la literatura científica a través de bases de datos tales como Web of Science, SCOPUS, LATINDEX y Google Académico. Una vez obtenida una información preliminar, se procedió a la redacción de los ítems de las escalas que configuraron el cuestionario. Después, se realizó un primer abordaje a través del pilotaje del cuestionario con una reducida muestra de estudiantes para valorar los tiempos y considerar las dudas semánticas que pudieran surgir. Se depuraron los aspectos gramaticales y de formato y se realizó la versión definitiva a través de una comisión formada por los miembros del grupo de investigación. Los cuestionarios fueron aplicados a través de un vínculo online proporcionado al alumnado o bien mediante encuestas impresas. La administración presencial del cuestionario tuvo una duración aproximada de 15-20 minutos, no imponiendo tiempo límite. Los miembros del equipo de investigación fueron los encargados de administrar el cuestionario y mecanizar los datos. Siguiendo los criterios éticos los participantes fueron informados del objetivo del cuestionario así como de la voluntariedad, anonimato y confidencialidad de los resultados.

\subsection{Participantes}


En el estudio participó una muestra representativa constituida por 765 estudiantes de una universidad del sureste español, de los cuáles 210 (28.48\%) cumplimentaron el QAEU en línea. El resto lo realizó presencialmente en el aula trasladándose para ello investigadores del grupo a dichas dependencias. En total, el $72.7 \%$ (556) fueron mujeres. La edad media del alumnado fue de 23.27 años $(D T=.81)$. En concreto, el $29.3 \%$ (224) tenía menos de 20 años, el 52.4\% (402) se ubicó en el intervalo de 20 a 24 años, el $11.2 \%$ (86) entre 25 y 29 años y el 6.9\% (53) 30 o más. Con relación al país, el 93.5\% (715) procedía de España y el $6.5 \%$ (50) de otros. La distribución de los participantes en función de los estudios que cursaban así como el curso del Grado $(N=637)$ se representan en la Figura 1 y 2 respectivamente. Como se puede apreciar en la Figura 1, la mayoría de los participantes son estudiantes de Ciencias Sociales y de la Educación y Ciencias de la Salud. Eran los de primer curso los que respondieron en mayor proporción, según se refleja en la Figura 2 .

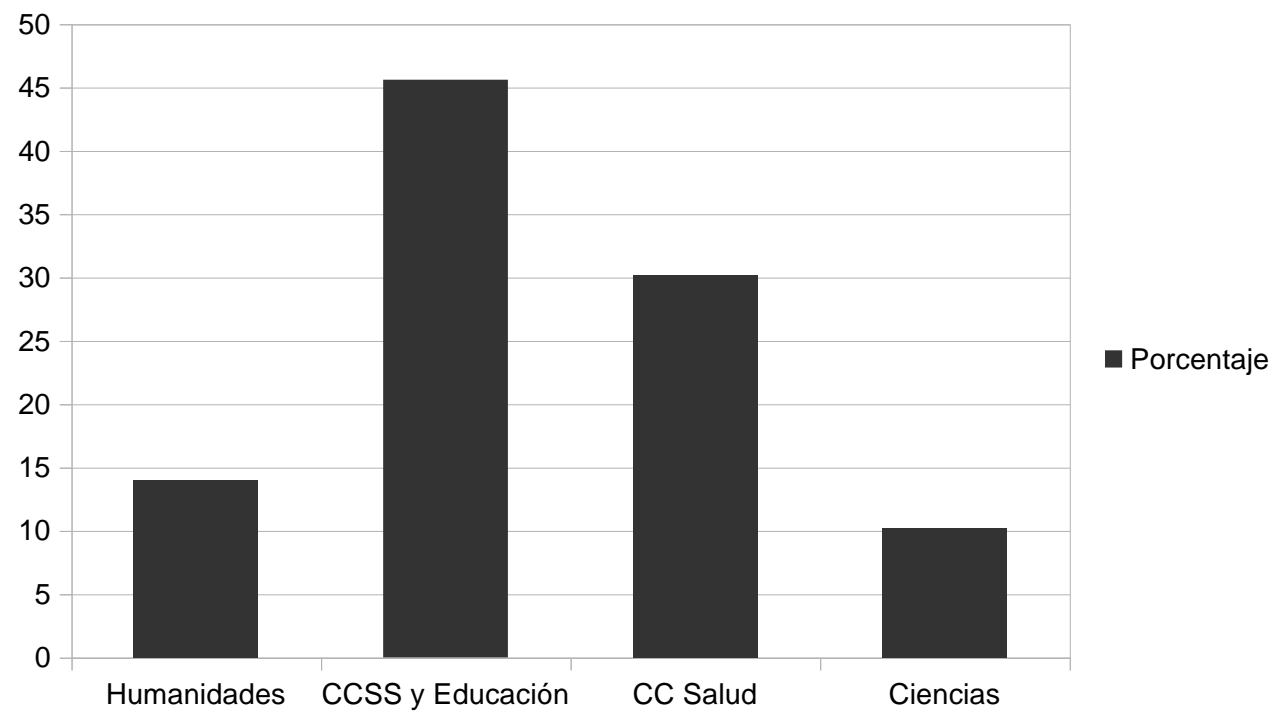

Figura 1. Distribución de participantes por facultades.

Nota. CCSS y Educación: Ciencias Sociales y de la Educación. CC Salud: Ciencias de la Salud.

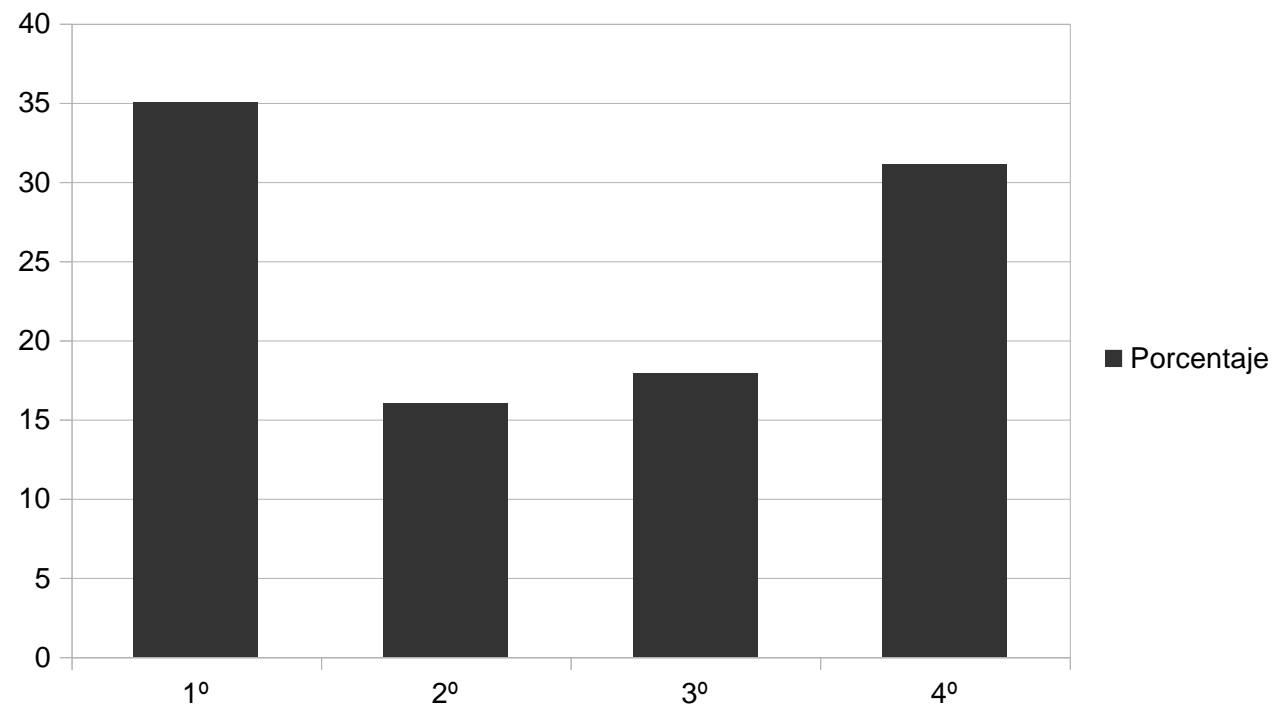

Figura 2. Distribución de los participantes en función del curso en el Grado. 


\subsection{Análisis de datos}

Se procedió al análisis de la información recogida usando los paquetes estadísticos SPSS versión 19 para el análisis sociodemográfico y R construcción del Modelo de Ecuaciones Estructurales, o «Structural Equations Model» (SEM), siguiendo la fundamentación teórica de Beaujean (2014). Dicho modelo fue utilizado para confirmar la existencia de los constructos descritos en los resultados. Con relación a las medidas de ajuste, se utilizó el Error Medio Cuadrático de Aproximación que mide la discrepancia entre el modelo y los datos (González-Montesinos y Backhoff, 2010). Asimismo, se usaron los siguientes índices de ajuste incremental (Hooper, Coughlan y Mullen, 2008): Índice de Ajuste No Normativo (NNFI), Índice de Ajuste Comparativo (CFI) e Îndice de Tucker-Lewis (TLI).

\section{Resultados}

Con respecto al análisis de la consistencia interna, en la Tabla 1 se exponen las puntuaciones obtenidas para cada una de las escalas del QAEU a través de Alpha de Cronbach. Todas las escalas obtuvieron una puntuación superior a .9 .

\section{Tabla 1}

Alpha de Cronbach por constructo

\begin{tabular}{lc}
\hline Factores & Alpha \\
\hline Como objeto de las agresiones & .95 \\
Como agente de las agresiones & .94 \\
Como observador de las agresiones & .96 \\
\hline
\end{tabular}

Se trata de valores altos para el Alpha de Cronbach en todos los factores. Las escalas de medida son fiables y existe consistencia interna de las cuestiones analizadas.

Al desarrollar el modelo de ecuaciones estructurales, se analizó el cuestionario diferenciando sus tres escalas: "como objeto de las agresiones", "como agente de las agresiones" y "como observador de las agresiones". En primer lugar, y con relación a la escala "como objeto de las agresiones", se estimaron los valores para los coeficientes de agresión directa $(A D)$, diferenciando entre: el tipo de agresión (ADt), quién la realizó (ADr), dónde (ADI), el momento (ADmm) y el motivo (ADmt). Igualmente, el modelo estimó los valores para los coeficientes de ciberacoso (CA) distinguiendo tipo (CAt), medio (CAv), momento en el que el sujeto se sintió a un lado del grupo (CAx), motivo (CAm), a quién se le comunicaron los hechos (CAn) y a quién se le proporcionó apoyo (CAy). En la Tabla 2 se muestran los resultados tras el uso de Lavaan (.5-23.1097), convergente normalmente después de 76 iteraciones, para una correlación simple de segundo orden (variante Mplus).

\section{Tabla 2}

Análisis para el SEM en la escala «como objeto de las agresiones»

\begin{tabular}{lcc}
\hline & Usado & Respuesta \\
\hline Número de observaciones & 513 & 765 \\
Estimador & DWLS & Robusto \\
Función mínima del test estadístico & 2787.432 & 1690.516 \\
Grados de libertad & 979 & 979 \\
Valor p (Chi Cuadrado) & .000 & .000 \\
Factor de corrección de escala & & 2.974 \\
Parámetro de turnos & & 753.395 \\
\hline
\end{tabular}


Dada la naturaleza de las variables, se optó por realizar una estimación robusta del estadístico $\chi^{2}$ a través del estimador DWLS ("Diagonal Weighted Least Squares", o estimador ponderado de mínimos cuadrados) tal como se expone en la Tabla 2. Por ende, existían diferencias entre la matriz de covarianza derivada de las variables observadas y la matriz reproducida por el modelo. Para poder afirmar que el modelo se ajustaba bien a los datos y dado que el estadístico de $x^{2}$ es sensible a las mínimas diferencias cuando la muestra es amplia, se calcularon otros índices de bondad de ajuste. Se utilizaron los índices de ajuste incremental NNFI, CFI y TLI, obteniéndose .978, .986 y .984 respectivamente. Valores por encima de .95 indicaron un buen ajuste. Además, el índice de bondad de ajuste Error Medio Cuadrático de Estimación ("Root Mean-Squared Error Aproximation", RMSEA) alcanzó una puntuación de .06 afirmándose que el modelo presentaba un ajuste razonable. En la Figura 3 se expone gráficamente lo que aportó cada variable al modelo, en función del grosor de la línea, distinguiendo entre agresión directa (agD) y ciberacoso (cbr). Todos los p-valores fueron significativos, tanto los provenientes de los ítems de agresividad directa (a) como de ciberacoso (c) por lo que todas las variables aportaron, en mayor o menor medida, al modelo.

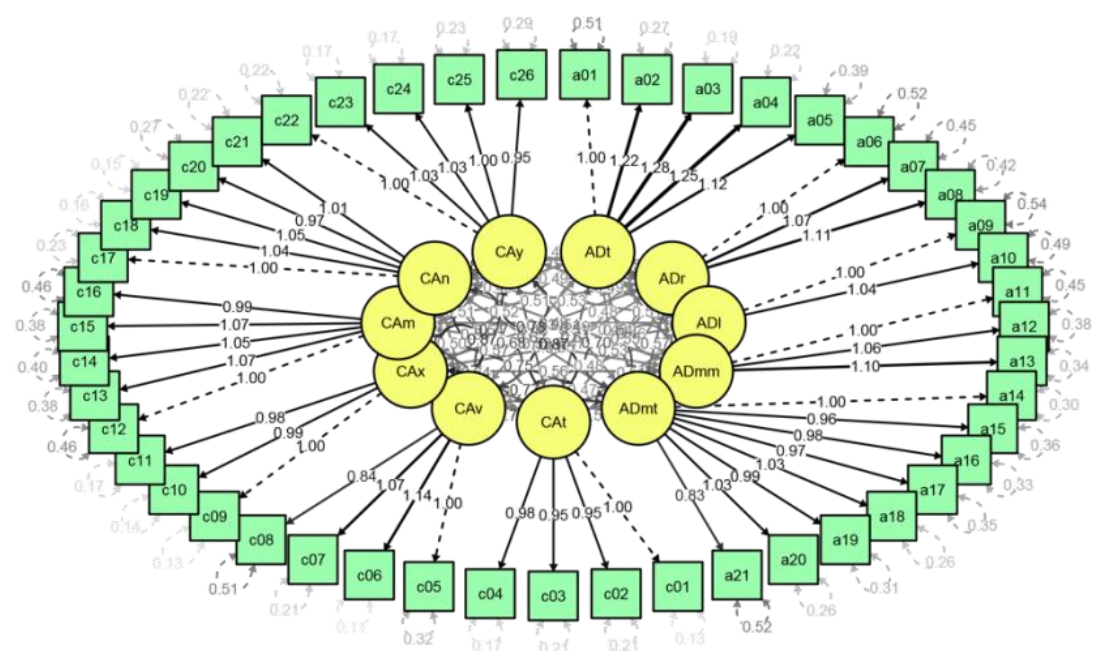

Figura 3. Modelo de Ecuaciones Estructurales. Constructo: Como objeto de las agresiones.

En segundo lugar, y con respecto a la escala "como agente de las agresiones", la variable dependiente fue el constructo agente de las agresiones tanto directas (AG) como mediante ciberacoso (CA) y las cuestiones las variables independientes referentes al tipo de agresión (AGt), hacia quién se dirigió (AGd), cuándo se produjo (AGmm) y la causa (AGmt). Igualmente, las siguientes cuestiones y variables dieron cuenta del ciberacosador como agente (CAR): qué acto se cometió (CARt), la vía (CARv), a quién se dejó al margen (CARx) y los motivos (CARm). En la Tabla 3 se muestran los resultados tras el uso de Lavaan (.5-23.1097), convergente normalmente después de 55 iteraciones, para una correlación simple de segundo orden (variante Mplus).

Tabla 3

Análisis para el SEM en la escala «como agente de las agresiones»

\begin{tabular}{lcc}
\hline & Usado & Respuesta \\
\hline Número de observaciones & 367 & 765 \\
Estimador & DWLS & Robusto \\
Función mínima del test estadístico & 288.938 & 448.306 \\
Grados de libertad & 463 & 436 \\
Valor p (Chi Cuadrado) & 1.000 & .332 \\
Factor de corrección de escala & & 3.061 \\
Parámetro de turnos & & 353.921 \\
\hline
\end{tabular}


En este caso, el $p$-valor fue de .332 por lo que no existían diferencias entre la matriz de covarianza derivada de las variables observadas y la matriz reproducida por el modelo o, lo que es lo mismo, que había un buen ajuste entre el modelo y los datos. Además, se obtuvo .994 en el NNFI, 1.000 en el Índice CFI y 1.004 en el Índice de Tucker-Lewis considerándose un buen ajuste. RMSEA fue 0 por lo que el ajuste fue excelente. En la Figura 4 se exponen gráficamente las relaciones que mantuvieron las variables del modelo referentes al tipo de ciberagresión (CARt) junto con el momento (CARm), exclusión (CARx) y vía (CARv) así como el tipo de agresión directa (AGt), dirección (AGd), momento (AGmm) y motivo (AGmt) con los ítems de la escala referentes a las agresiones directas (a) y ciberacoso (c).

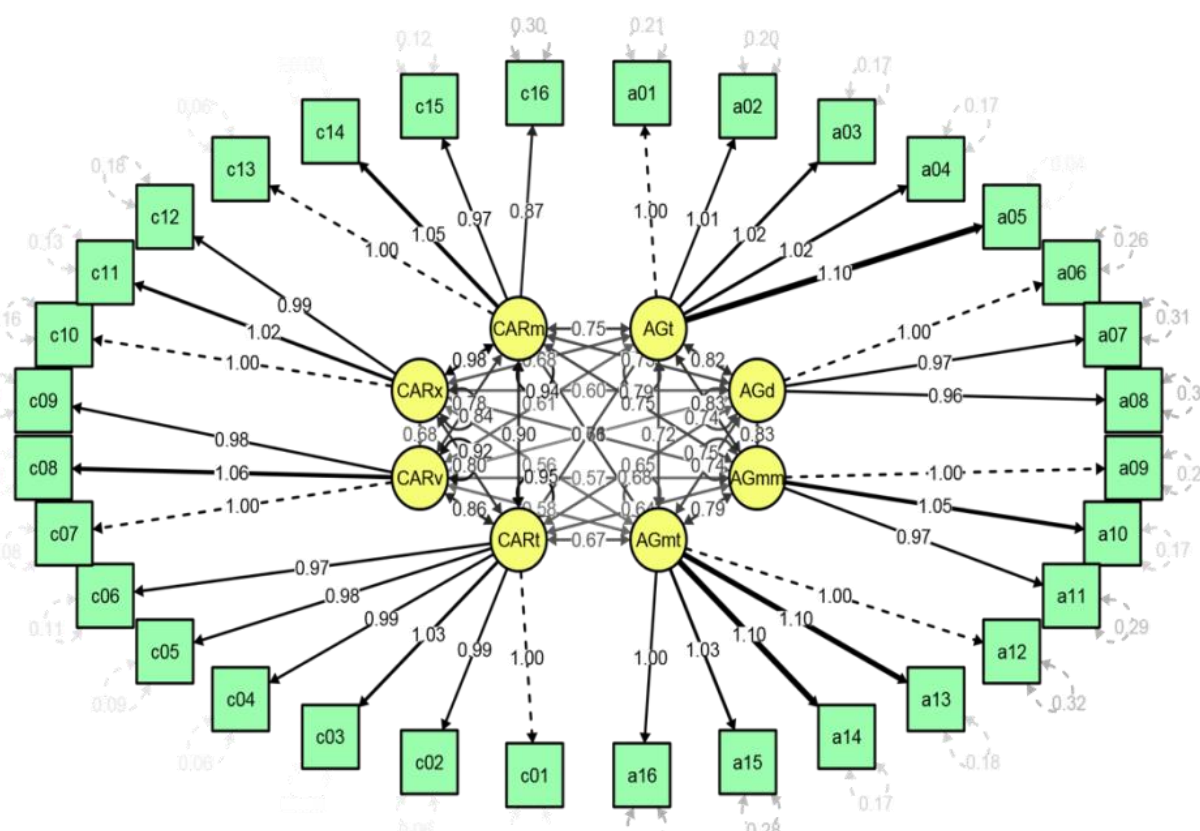

Figura 4. Modelo de Ecuaciones Estructurales. Constructo: Como agente de las agresiones.

En tercer y último lugar, en la escala "como observador de las agresiones", la variable dependiente fue el constructo "observador» (OB) y las cuestiones las variables independientes relativas al tipo de agresión directa observada (OBt), hacia quién iba dirigida (OBd), en qué momento (Obmm) y los motivos (OBmt). Igualmente, y con relación al observador de ciberagresiones (OBC), se hizo una distinción en función del tipo $(\mathrm{OBCt})$, vía $(\mathrm{OBCv})$, quiénes son los excluidos (OBCx), causas $(\mathrm{OBCm})$, a quién se informó $(\mathrm{OBCn})$ y a quién se solicitó ayuda (OBCy). En la Tabla 4 se muestran los resultados tras el uso de Lavaan (.5-23.1097), convergente normalmente tras 83 iteraciones, para una correlación simple de segundo orden (variante Mplus).

Tabla 4

Análisis para el SEM en la escala «como observador de las agresiones»

\begin{tabular}{lcc}
\hline & Usado & Respuesta \\
\hline Número de observaciones & 493 & 765 \\
Estimador & DWLS & Robusto \\
Función mínima del test estadístico & 1352.456 & 1168.690 \\
Grados de libertad & 815 & 815 \\
Valor p (Chi Cuadrado) & .000 & .000 \\
Factor de corrección de escala & & 2.517 \\
Parámetro de turnos & & 631.297 \\
\hline
\end{tabular}


Tal como se expone en la Tabla 4, el estimador fue DWLS con estadístico 1352.456 (estimación robusta 1168.690), con 815 grados de libertad y un p-valor significativo (<.05), y la naturaleza de las variables, llevaron a realizar análisis adicionales para asegurar el ajuste entre el modelo y los datos. Concretamente, los índices de ajuste incremental se situaron en .994 en el caso del Índice NNFI, .998 en el CFI y .997 en el ITL, indicando un ajuste excelente ( $p>.95)$. RMSEA obtuvo un ajuste bueno (RMSEA=.037). En la Figura 5 se expone de forma gráfica lo que aportó cada variable al modelo, señalándose tanto los ítems referentes al observador de la agresión (ob) como del ciberacoso (o). Todos los $p$-valores fueron significativos por lo que todas las variables influyeron en el modelo.

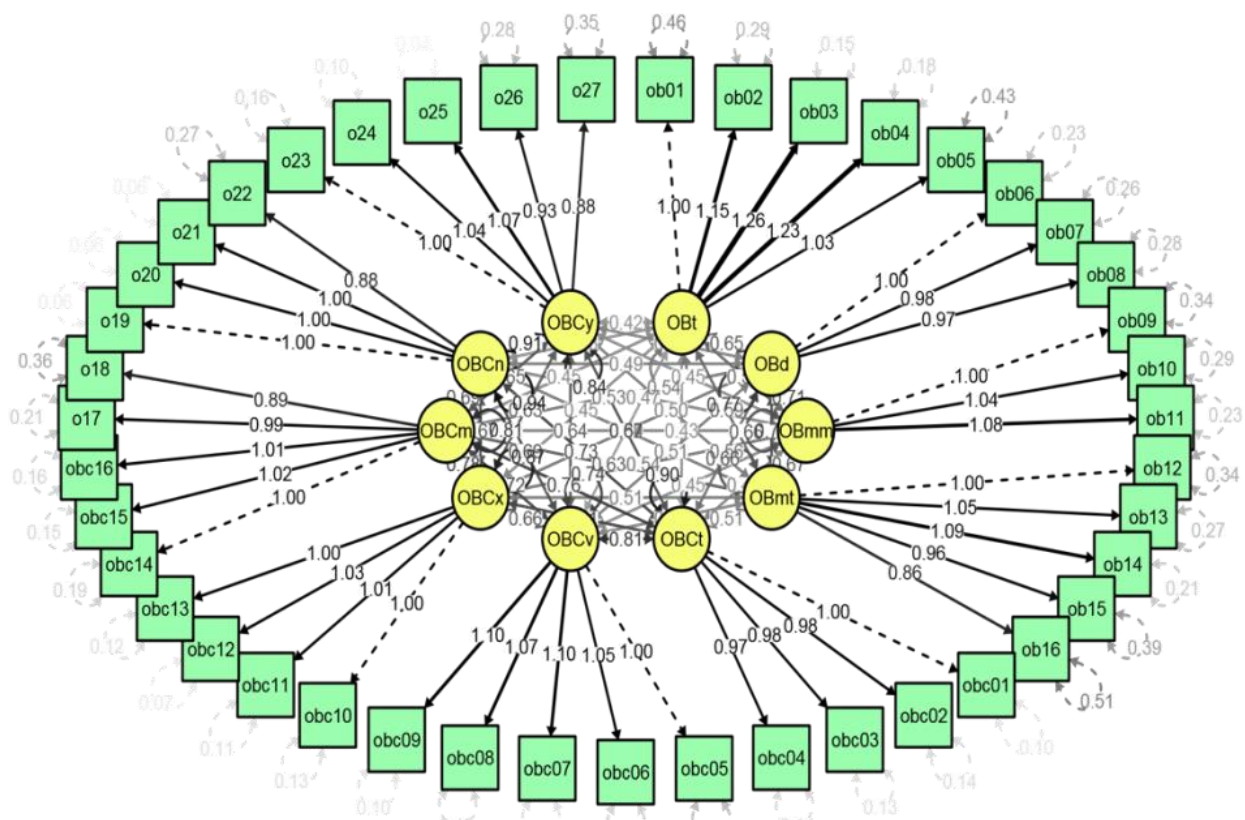

Figura 5. Modelo de Ecuaciones Estructurales. Constructo: Como observador de las agresiones.

\section{Discusión}

Con respecto al primer objetivo del estudio referente al análisis de la consistencia interna, se obtienen valores muy altos para el Alpha de Cronbach en todos los factores: "como objeto de las agresiones" (.95), "como agente de las agresiones" (.94) y "como observador de las agresiones" (.96). Previamente, el Cuestionario para Estudiantes (Hoyos et al, 2009) arrojó un Alpha de Cronbach global de .81 en universitarios (Hoyos et al, 2012) y el "Cyberbullying. Screening de acoso entre iguales" de Garaigordobil (2013) obtuvo en sus escalas valores que oscilaron entre .80 y .91 . Por ende, es posible afirmar que las escalas de medidas del QAEU son fiables y existe una alta consistencia interna en las cuestiones analizadas. Este dato apoya el uso del QAEU para detectar el acoso y ciberacoso en contextos universitarios.

En segundo lugar, y con respecto al segundo objetivo de la investigación, se demuestra que el análisis de ecuaciones estructurales realizado permite validar la existencia de los constructos estudiados en el QAEU. En todos ellos los índices de ajuste incremental y el RMSEA permiten concluir un ajuste muy bueno/excelente entre los datos y el modelo siguiendo a Hooper et al. (2008) y a González-Montesinos y Backhoff (2010).

Asimismo, es posible afirmar que tanto el cuestionario de Garaigordobil (2013) como el QAEU permiten detectar si el alumnado es víctima, agresor u observador si bien el segundo se adapta al vocabulario universitario. La comunidad universitaria precisa de mayor formación en este ámbito para su detección precoz (Alonso-Martín, 2009), y el QAEU contribuye a este objetivo.

Otra aportación del QAEU es la inclusión de un mayor número de variables que profundizan en elementos como los motivos que llevan al sujeto a ubicarse en uno de los tres roles y a quién informa y solicita apoyo en la Universidad española. Garaigordobil (2015) recurre al análisis factorial de las escalas del cuestionario de origen obteniendo también una estructura evidente de los constructos de acoso y ciberacoso considerando 15 ítems para cada rol mientras que el QAEU aporta mayor información al elevar el número de ítems. En este sentido, el QAEU introduce en un solo instrumento, entre otros elementos, la evaluación de las vías de 
interacción o comunicación (Ortega-Ruiz et al., 2016), el origen o procedencia del agresor, agredido u observador (Perry y Blincoe, 2015) y los roles (Garaigordobil, 2015; Martínez-Monteagudo et al., 2019) tanto para acoso como ciberacoso.

Con relación a la aplicabilidad del QAEU y contribución al campo de estudio, es posible afirmar que este cuestionario permite a los profesionales obtener información fiable y válida sobre el acoso y ciberacoso. El cuestionario QAEU puede ser utilizado para analizar la prevalencia del fenómeno, su presencia, la distinción por roles, entre otros aspectos, de modo que puedan desarrollarse programas proactivos y reactivos como los planteados por McCoy, Englander y Parti (2018) al describir programas para entrenar a futuros educadores para prevenir el acoso. En términos de Beaujean (2014), se trata de un instrumento estadísticamente robusto. Simultáneamente, el QAEU es sencillo de administrar, de gran rapidez y disminuye el fenómeno de deseabilidad social. La aplicación de este cuestionario ha permitido comprobar que tanto hombres como mujeres están involucrados en casos de acoso y que éste varía en función del rango de edad, habiendo mayor implicación como agentes de las agresiones entre aquellos estudiantes universitarios entre 20 y 24 años en relación con los mayores de 30 (Méndez, Ruiz-Esteban, Martínez y Cerezo, 2019a).

\section{Conclusiones}

En definitiva, el QAEU es un instrumento psicométrico para medir acoso y ciberacoso en estudiantes hispanohablantes universitarios con una alta consistencia interna, la cual oscila entre .94 y .96, diferenciándose tres dimensiones: "como objeto de las agresiones", "como agente de las agresiones" y "como observador de las agresiones". Dichas dimensiones han sido validadas mediante un modelo de ecuaciones estructurales con un ajuste muy bueno/excelente entre los datos y el modelo.

Con respecto a las limitaciones del estudio, debido a que el cuestionario se ha diseñado, pilotado y puesto en marcha con población universitaria, la extrapolación de los resultados a otros colectivos debe ser realizada con precaución. Al igual que se postula en otras investigaciones (Álvarez-García, Barreiro-Collazo, Núñez y Dobarro, 2016; Ortega-Ruiz et al., 2016), la validación del instrumento en otros segmentos socioculturales y de edad aportaría información relevante y aumentaría la representatividad de la muestra. Por otro lado, los resultados del modelo en algunas de sus escalas podrían llevar a plantearse una sobreestimación del mismo. Con relación a futuras líneas de investigación, se estima pertinente internacionalizar el cuestionario y realizar estudios comparativos y longitudinales. Con relación a la estructura de los constructos, a pesar de existir evidencias de una relación entre acoso y ciberacoso (Ortega-Ruiz et al., 2016), cabe considerar que ambos diverjan hasta constituir representaciones de fenómenos distintos. En definitiva, es preciso seguir investigando para lograr una mayor comprensión de la temática (Garaigordobil, 2015) y sensibilizar a la comunidad educativa (Méndez, Ruiz-Esteban, Martínez y Cerezo, 2019b).

\section{Referencias}

Alonso-Martín, P. (2009). La visión del fenómeno bullying en alumnos del Curso de Aptitud Pedagógica en los años 2005/06 y 2006/07: semejanzas y diferencias. Estudios sobre Educación, 17, 125-144.

https://doi.org/10.15581/004.17.125-144

Álvarez-García, D., Barreiro-Collazo, A., Núñez, J. C. y Dobarro, A. (2016). Validity and reliability of the Cyberaggression Questionnaire for Adolescents (CYBA). The European Journal of Psychology Applied to Legal Context, 8 , 69-77.

https://doi.org/10.1016/j.ejpal.2016.02.003

Avilés, J. M. (2009). Ciberbullying. Diferencias entre el alumnado de secundaria. Boletín de Psicología, 96, 79-96.

Recuperado de: https://goo.gl/eP45w6

Beaujean, A. (2014). Latent Variable Modeling Using R: A Step-by-Step Guide. New York, NY: Routledge.

Bender, D. y Lösel, F. (2011). Bullying at school as a predictor of delinquency, violence and other anti-social behaviour in adulthood. Criminal Behaviour and Mental Health, 21, 99-106.

Cerezo, F., Sánchez, C., Ruiz-Esteban, C. y Arense, J. J. (2015). Adolescents and Preadolescents' Roles on Bullying, and Its Relation with Social Climate and Parenting Styles. Revista de Psicodidáctica, 20, 139-155.

https://doi.org/10.1387/RevPsicodidact.11097

Defensor del Pueblo (2000). Informe sobre violencia escolar: el maltrato entre iguales en la educación secundaria obligatoria. Madrid: Publicaciones de la Oficina del Defensor del Pueblo. Recuperado de: https://goo.gl/4L8Me9

Faucher, C., Jackson, M. y Cassidy, W. (2014). Cyberbullying among university students: Gendered experiences, impacts, and perspectives. Education Research International, 1-10.

https://doi.org/10.1155/2014/698545

Garaigordobil, M. (2013). Cyberbullying. Screening de acoso entre iguales. Madrid: TEA. 
Garaigordobil, M. (2015). Ciberbullying en adolescentes y jóvenes del País Vasco: Cambios con la edad. Anales de Psicología, 31, 1069. https://doi.org/10.6018/analesps.31.3.179151

Gascón-Cánovas, J. J., Russo, J. R., Cózar, A. y Heredia, J. M. (2016). Adaptación cultural al español y baremación del Adolescent Peer Relations Instrument (APRI) para la detección de la victimización por acoso escolar: Estudio preliminar de las propiedades psicométricas. Anales de Pediatría, 87, 9-17. https://doi.org/10.1016/j.anpedi.2015.12.003

Gómez-Ortiz, O., Del Rey, R., Romera, E. M. y Ortega-Ruiz, R. (2015). Los estilos educativos paternos y maternos en la adolescencia y su relación con la resiliencia, el apego y la implicación en acoso escolar. Anales de Psicología, 31, 979-989.

https://doi.org/10.6018/analesps.31.3.180791

Gómez-Ortiz, O., Romera-Félix, E. M. y Ortega-Ruiz, R. (2017). Multidimensionalidad de la competencia social: medición del constructo y su relación con los roles del bullying. Revista de Psicodidáctica, 22, 37-44. https://doi.org/10.1016/S1136-1034(17)30042-4

González-Montesinos, M. J. y Backhoff, E. (2010). Validación de Un Cuestionario de Contexto Para Evaluar Sistemas Educativos Con Modelos de Ecuaciones Estructurales. Relieve, 16, 1-17.

Halpern, D., Piña, M. y Vásquez, J. (2017). Loneliness, personal and social well-being: towards a conceptualization of the effects of cyberbullying / Soledad, bienestar social e individual: hacia una conceptualización de los efectos del cyberbullying. Cultura y Educación, 29(4), 703-727.

https://doi.org/10.1080/11356405.2017.1370818

Herrera-López, M., Romera, E. y Ortega-Ruiz, R. (2017). Bullying y cyberbullying en Colombia; coocurrencia en adolescentes escolarizados. Revista Latinoamericana de Psicología, 49, 163-172.

https://doi.org/10.1016/j.rlp.2016.08.001

Hooper, D., Coughlan, J. y Mullen, M. (2008). Structural Equation Modelling: Guidelines for Determining Model Fit. Electronic Journal of Business Research Methods, 6, 53-60.

Hoyos, O. L. R., Llanos M. M. y Valega, S. J. (2012). El maltrato entre iguales por abuso de poder en el contexto universitario: incidencia, manifestaciones y estrategias de solución. Universitas Psychologica, 11, 793-802.

Hoyos, O. L. R., Romero, L., Valega, S. J. y Molinares, C. (2009). El maltrato entre iguales por abuso de poder y exclusión social en estudiantes de una universidad privada de la ciudad de Barranquilla. Pensamiento Psicológico, 6 , 109-125.

Kokkinos, C., Antoniadou, N. y Markos, A. (2014). Cyber-bullying: An investigation of the psychological profile of university student participants. Journal of Applied Developmental Psychology, 35, 204-214. https://doi.org/10.1016/j.appdev.2014.04.001

Kopecký, K. (2013). Cyberbullying and other risks of Internet communication focused on university students. Procedia - Social and Behavioral Science, 112, 260-269.

https://doi.org/10.1016/i.sbspro.2014.01.1163

López, M. G. (2016). Validación de un instrumento para medir el acoso escolar en estudiantes mexicanos. Enseñanza e Investigación en Psicología, 21, 291-299.

López, M. G. (2017). Influencia del clima escolar y familiar en el acoso escolar y cibernético de universitarios. Revista Mexicana de Investigación en Psicología, 9(1), 31-44.

McCoy, M. K., Englander, E. K. y Parti, K. (2018). A model for providing bullying prevention programs to K-12 education while training future educators. Reducing Cyberbullying in Schools, International Evidence-Based Best Practices, 109-124.

https://doi.org/10.1016/B978-0-12-811423-0.00008-0

Magaz, A., Chorot, P., Santed, M., Valiente, R. y Sandín, B. (2016). Evaluación del bullying como victimización:

Estructura, fiabilidad y validez del Cuestionario de Acoso entre Iguales (CAI). Revista de Psicopatología y Psicología Clínica, 21, 77-95.

https://doi.org/10.5944/rppc.vol.21.num.2.2016.16990

Martínez, J. P., Méndez, I. y Cerezo, F. (2011). Concienciación de la violencia escolar en el alumnado: una aproximación práctica. En UPTC (Ed.), Congreso Internacional de Innovación Docente (pp. 1233-1242). Cartagena, España.

Martínez-Monteagudo, M.C., Delgado, B., Inglés, C. J. y García-Fernández, J. M. (2019). Cyberbullying in the university setting. Relationship with family environment and emotional intelligence. Computers in Human Behavior, 91, 220-225.

https://doi.org/10.1016/j.chb.2018.10.002

Méndez, I. y Cerezo, F. (2010). Bullying y factores de riesgo para la salud en estudiantes de secundaria. European Journal of Education and Psychology, 3, 209-218.

https://doi.org/10.1989/ejep.v3i2.61

Méndez, I., Ruiz-Esteban, C., Martínez, J.P. y Cerezo, F. (2019a). Acoso escolar en el ámbito universitario. Behavioral Psychology/Psicología Conductual, 27(1), 55-68. 
Méndez, I., Ruiz-Esteban, C., Martínez, J.P. y Cerezo, F. (2019b). Ciberacoso según características sociodemográficas y académicas en estudiantes universitarios. Revista Española de Pedagogía, 77(273), $261-276$. doi:10.22550/REP77-02-2019-06

Montero, I. y León, O. G. (2007). A guide for naming research studies in Psychology. International Journal of Clinical and Health Psychology, 7, 847-862. Recuperado de: https://goo.gl/YrZJJU

Ortega-Ruiz, R., Del Rey, R. y Casas, J. A. (2016). Evaluar el bullying y el cyberbullying validación española del EBIP$Q$ y del ECIP-Q. Psicología Educativa, 22, 71-79.

https://doi.org/10.1016/j.pse.2016.01.004

Perry, A. y Blincoe, S. (2015) Bullies and Victims in Higher Education. Journal of Bullying and Social Aggression, 1(1). Romera, E., Cano, J., García-Fernández, C. y Ortega-Ruiz, R. (2016). Cyberbullying: Social Competence, Motivation and Peer Relationships. [Cyberbullying: competencia social, motivación y relaciones entre iguales]. Comunicar, 48, 7179.

https://doi.org/10.3916/C48-2016-07

Sinkkonen, H.M., Puhakka, H. y Meriläinen, M. (2014) Bullying at a university: Students' experiences of bullying.

Studies in Higher Education, 39, 153-165.

https://doi.org/10.1080/03075079.2011.649726

Trujillo, J. J. y Romero-Acosta, K. (2016). Variables que evidencian el bullying en un contexto universitario. Revista Encuentros, 14, 41- 54.

https://doi.org/10.15665/re.v14i1.668

Vera, C. Y., Vélez, C. M. y García, H. I. (2017). Medición del bullying escolar: Inventario de instrumentos disponibles en idioma español. PSIENCIA. Revista Latinoamericana de Ciencia Psicológica, 9, 1-16.

https://doi.org/10.5872/psiencia/9.1.31 


\section{Anexo I}

Cuestionario sobre Acoso entre Estudiantes Universitarios (QAEU)

\section{PRESENTACIÓN}

Algunas veces, durante el trabajo en grupo o en el aula, surgen conflictos donde la exclusión, el menosprecio, el acoso directo (bullying) o a través del móvil o internet (ciberbullying), se manifiestan entre los compañeros.

El grupo de investigación EIPSED de la Universidad de Murcia está estudiando la incidencia de esta problemática en nuestro entorno, mediante un sondeo de opinión entre los estudiantes de esta universidad. Es por ello que solicitamos tu colaboración contestando a este cuestionario en el que se pregunta sobre esta problemática desde tres perspectivas diferentes: como víctima, como agresor y como observador. Para responder, una vez leída la pregunta, elige la opción que mejor se ajuste a lo que tú percibes, haciendo una cruz en la casilla correspondiente o bien rellenando la respuesta en la hoja adjunta.

\section{Todas las respuestas son anónimas y confidenciales.}

\section{DATOS SOCIODEMOGRÁFICOS}

1. Edad:

\begin{tabular}{|ll|l|}
\hline a. & Menos de 20 años & \\
\hline b. & Entre 20-24 & \\
\hline c. & Entre 25-29 & \\
\hline d. 30 o más años & \\
\hline
\end{tabular}

2. Sexo:

\begin{tabular}{|l|l|}
\hline a. Varón & \\
\hline b. Mujer & \\
\hline
\end{tabular}

3. Facultad donde cursas o cursaste el grado o licenciatura:

\begin{tabular}{|ll|l|}
\hline a. & $\begin{array}{l}\text { Humanidades (Bellas Artes, Geografía, Historia, Lengua, Leguas clásicas, Filosofía, } \\
\text { y otras). }\end{array}$ & \\
\hline b. $\begin{array}{l}\text { Ciencias Sociales y de la Educación (Derecho, Económicas, Trabajo Social, } \\
\text { Educación y otras). }\end{array}$ & \\
\hline c. & Ciencias de la Salud (Medicina, Enfermería, Psicología, Óptica, Fisioterapia y otras). & \\
\hline d. & Ciencias (Física, Química, Biología, Informática, Ingenierías y otras). & \\
\hline
\end{tabular}

4. Actualmente estoy cursando:

\begin{tabular}{|ll|l|}
\hline a. & Grado & \\
\hline b. $\quad$ Máster & \\
\hline c. & Otros estudios & \\
\hline
\end{tabular}

6. País de procedencia: a. España
5. Si estudias un Grado indica el curso

(en caso negativo pasa al ítem 6):

\begin{tabular}{|ll|l|}
\hline a. & $1^{\circ}$ & \\
\hline b. & $2^{\circ}$ & \\
\hline c. & $3^{\circ}$ & \\
\hline d. & $4^{\circ}$ & \\
\hline
\end{tabular}


Bloque 1. COMO OBJETO DE LAS AGRESIONES

Según tu experiencia, desde que empezaste los estudios universitarios

7. En alguna ocasión he sufrido agresión directa:

a

SI b NO

\begin{tabular}{|c|c|c|c|c|}
\hline & $\begin{array}{c}\text { NUNCA } \\
\text { a }\end{array}$ & $\begin{array}{c}\text { ALGUNAS } \\
\text { VECES } \\
\text { b }\end{array}$ & $\begin{array}{c}\text { A } \\
\text { MENUDO } \\
\text { c }\end{array}$ & $\begin{array}{c}\text { SIEMPRE } \\
\text { d }\end{array}$ \\
\hline \multicolumn{5}{|l|}{ ADt. He sufrido agresión de tipo: } \\
\hline \multicolumn{5}{|l|}{ ad01. Física } \\
\hline \multicolumn{5}{|l|}{ ad02. Verbal (insultos, amenazas, etc.) } \\
\hline \multicolumn{5}{|l|}{ ad03. Social (exclusión, aislamiento, etc.) } \\
\hline \multicolumn{5}{|l|}{ ad04. Psicológica (menosprecio, etc.) } \\
\hline \multicolumn{5}{|l|}{ ad05. Sexual } \\
\hline \multicolumn{5}{|l|}{ ADr. Las realizó: } \\
\hline \multicolumn{5}{|l|}{ ad06. Un/os chico/s } \\
\hline \multicolumn{5}{|l|}{ ad07. Una/s chica/s } \\
\hline \multicolumn{5}{|l|}{ ad08. Un grupo de chicos y chicas } \\
\hline \multicolumn{5}{|l|}{ ADl. Se produjo en: } \\
\hline \multicolumn{5}{|l|}{ ad09. Clase } \\
\hline \multicolumn{5}{|l|}{ ad10. Fuera del aula } \\
\hline \multicolumn{5}{|l|}{ ADmm. En qué momento: } \\
\hline \multicolumn{5}{|l|}{ ad11. Durante el trabajo en grupo } \\
\hline \multicolumn{5}{|l|}{ ad12. Durante el tiempo de clase } \\
\hline \multicolumn{5}{|l|}{ ad13. En cualquier momento } \\
\hline \multicolumn{5}{|l|}{ ADmt. Cuál fue el motivo : } \\
\hline \multicolumn{5}{|l|}{$\begin{array}{l}\text { ad14. Por mis creencias religiosas, políticas, } \\
\text { etc. }\end{array}$} \\
\hline \multicolumn{5}{|l|}{ ad15. Por mi orientación sexual } \\
\hline \multicolumn{5}{|l|}{ ad16. Por mi aspecto físico } \\
\hline \multicolumn{5}{|l|}{ ad17. Por cuestiones académicas } \\
\hline \multicolumn{5}{|l|}{$\begin{array}{l}\text { ad18. Me limitaron participar en la } \\
\text { toma de decisiones académicas }\end{array}$} \\
\hline \multicolumn{5}{|l|}{$\begin{array}{l}\text { ad19. Me limitaron información } \\
\text { relevante de tareas de clase o de grupo }\end{array}$} \\
\hline $\begin{array}{l}\text { ad20. Me limitaron otras } \\
\text { informaciones (becas, ayudas, etc.) }\end{array}$ & & & & \\
\hline ad21. No lo sé & & & & \\
\hline
\end{tabular}

29. En alguna ocasión he sufrido ciberacoso (Me han agredido mediante el móvil o internet):
$\mathrm{a}$ SI
$\mathrm{b} \mathrm{NO}$ 


\begin{tabular}{|c|c|c|c|c|}
\hline & $\begin{array}{c}\text { NUNCA } \\
\text { a }\end{array}$ & $\begin{array}{c}\text { ALGUNAS } \\
\text { VECES } \\
\text { b }\end{array}$ & $\begin{array}{c}\text { A } \\
\text { MENUDO } \\
\text { c }\end{array}$ & $\begin{array}{c}\text { SIEMPRE } \\
\text { d }\end{array}$ \\
\hline \multicolumn{5}{|l|}{ CAt. De qué tipo: } \\
\hline \multicolumn{5}{|l|}{ ca01. Verbal (llamadas, mensajes, etc.) } \\
\hline \multicolumn{5}{|l|}{ ca02. Social } \\
\hline \multicolumn{5}{|l|}{ ca03. Psicológico } \\
\hline \multirow{2}{*}{\multicolumn{5}{|c|}{$\begin{array}{l}\text { ca04. Acoso sexual } \\
\text { CAv. través de: }\end{array}$}} \\
\hline & & & & \\
\hline \multicolumn{5}{|l|}{ ca05. Llamadas } \\
\hline \multicolumn{5}{|l|}{ ca06. Mensaje de texto } \\
\hline \\
\hline \multicolumn{5}{|l|}{$\begin{array}{l}\text { ca08. La difusión de fotos o vídeos privados } \\
\text { sin permiso }\end{array}$} \\
\hline \multicolumn{5}{|l|}{ CAx. Me he sentido excluido/a del grupo: } \\
\hline \\
\hline \multicolumn{5}{|l|}{$\begin{array}{l}\text { ca10. En información relevante de tareas de } \\
\text { clase o de grupo }\end{array}$} \\
\hline \multicolumn{5}{|l|}{$\begin{array}{l}\text { ca11. Otras informaciones (becas, ayudas, } \\
\text { etc.) }\end{array}$} \\
\hline \multicolumn{5}{|l|}{ CAm. Las agresiones se producen por: } \\
\hline \multicolumn{5}{|l|}{ ca12. Mis creencias religiosas, políticas, etc. } \\
\hline \multicolumn{5}{|l|}{ ca13. Mi orientación sexual } \\
\hline \multicolumn{5}{|l|}{ ca14. Mi aspecto físico } \\
\hline \multicolumn{5}{|l|}{ ca15. Cuestiones académicas } \\
\hline ca16. No lo sé & & & & \\
\hline \multicolumn{5}{|c|}{ CAn. Cuando he sido acosado/a he informado a: } \\
\hline \multicolumn{5}{|l|}{ ca17. Algún compañero/a } \\
\hline \multicolumn{5}{|l|}{ ca18. Algún profesor/a } \\
\hline \multicolumn{5}{|l|}{ ca19. Algún responsable de la institución } \\
\hline \multicolumn{5}{|l|}{ ca20. Otros } \\
\hline \multirow{2}{*}{\multicolumn{5}{|c|}{$\begin{array}{l}\text { ca21. No he informado } \\
\text { CAv. Cuando he sido acosado/a he pedido avuda a: }\end{array}$}} \\
\hline & & & & \\
\hline \multicolumn{5}{|l|}{ ca22. Algún compañero/a } \\
\hline \multicolumn{5}{|l|}{ ca23. Algún profesor/a } \\
\hline \multirow{2}{*}{\multicolumn{5}{|c|}{$\begin{array}{l}\text { ca24. Algún responsable de la institución } \\
\text { ca25. Otros }\end{array}$}} \\
\hline & & & & \\
\hline ca26. No he pedido ayuda & & & & \\
\hline
\end{tabular}

Bloque 2. COMO AGENTE DE LAS AGRESIONES

Según tu experiencia, desde que empezaste los estudios universitarios

56. En alguna ocasión he acosado a algún compañero o compañera:

$\mathrm{a} \mathrm{SI} \quad \mathrm{b} \mathrm{NO}$




\begin{tabular}{|c|c|c|c|c|}
\hline & $\begin{array}{c}\text { NUNCA } \\
\text { a }\end{array}$ & $\begin{array}{c}\text { ALGUNAS } \\
\text { VECES } \\
\text { b }\end{array}$ & $\begin{array}{c}\text { A } \\
\text { MENUDO } \\
\text { c }\end{array}$ & $\begin{array}{c}\text { SIEMPRE } \\
\text { d }\end{array}$ \\
\hline \multicolumn{5}{|l|}{ AGt. De forma: } \\
\hline \multicolumn{5}{|l|}{ ag01. Física } \\
\hline \multicolumn{5}{|l|}{ ag02. Verbal (insultos, amenazas, etc.) } \\
\hline \multicolumn{5}{|l|}{ ag03. Social (exclusión, aislamiento, etc.) } \\
\hline \multicolumn{5}{|l|}{ ag04. Psicológica (menosprecio, etc.) } \\
\hline \multicolumn{5}{|l|}{ ag05. Sexual } \\
\hline \multicolumn{5}{|l|}{ AGd. Las agresiones las dirijo a: } \\
\hline \multicolumn{5}{|l|}{ ag06. Un/os chico/s } \\
\hline \multicolumn{5}{|l|}{ ag07. Una/s chica/s } \\
\hline \multicolumn{5}{|l|}{ ag08. Un grupo de chicos y chicas } \\
\hline \multicolumn{5}{|l|}{ AGmm. Las agresiones se producen: } \\
\hline \multicolumn{5}{|l|}{ ag09. Durante el trabajo en grupo } \\
\hline \multicolumn{5}{|l|}{ ag10. Durante la clase } \\
\hline \multicolumn{5}{|l|}{ ag11. En cualquier momento } \\
\hline \multicolumn{5}{|l|}{ AGmt. Las agresiones se producen por: } \\
\hline \multicolumn{5}{|l|}{ ag12. Las creencias (religiosas, políticas, etc.) } \\
\hline \multicolumn{5}{|l|}{ ag13. La orientación sexual } \\
\hline \multicolumn{5}{|l|}{ ag14. El aspecto físico } \\
\hline ag15. Cuestiones académicas & & & & \\
\hline ag16. No lo sé & & & & \\
\hline
\end{tabular}

73. En alguna ocasión he realizado ciberacoso (Agredido a otros mediante el móvil o Internet):

$$
\mathrm{a} \text { SI } \mathrm{b} \mathrm{NO}
$$

\begin{tabular}{|c|c|c|c|c}
\hline NUNCA & $\begin{array}{c}\text { ALGUNAS } \\
\text { VECES } \\
\text { a }\end{array}$ & $\begin{array}{c}\text { A } \\
\text { MENUO } \\
\text { c }\end{array}$ & $\begin{array}{c}\text { SIEMPRE } \\
\mathbf{d}\end{array}$ \\
\hline
\end{tabular}

CARt. A través de:

car01. Mensajes ofensivos

car02. Mediante llamadas ofensivas

car03. He difundido fotos o vídeos sin permiso

car04. He chantajeado o amenazado

car05. He acosado sexualmente

car06. He difamado

CARv. He utilizado:

car07. Mensajería instantánea

car08. Mensaje de texto

car09. Redes sociales

CARx. He excluido a alguien:

car10. Del grupo de amigos

car11. Del grupo de trabajo

\begin{tabular}{|l|l|l|l|}
\hline & & & \\
\hline & & & \\
\hline & & & \\
\hline & & & \\
\hline & & & \\
\hline & & & \\
\hline
\end{tabular}

\begin{tabular}{l|l|l|l}
\hline & & & \\
\hline & & & \\
\hline & & & \\
\hline
\end{tabular}

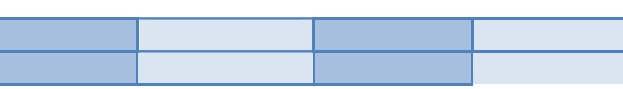


car12. Del grupo aula

CARm. Las agresiones se producen por:

car13. Las creencias religiosas, políticas, etc.

car14. La orientación sexual

car15. El aspecto físico

car16. Cuestiones académicas

car17. Otros motivos

Bloque 3. COMO OBSERVADOR/A DE LAS AGRESIONES

Según tu experiencia, desde que empezaste los estudios universitarios

91. En alguna ocasión, he visto agredir a otro estudiante:

$\mathrm{a} \quad \mathrm{SI} \quad \mathrm{b} \mathrm{NO}$

\begin{tabular}{c|c|c|c|c}
\cline { 2 - 5 } & NUNCA & $\begin{array}{c}\text { ALGUNAS } \\
\text { VECES } \\
\text { b }\end{array}$ & $\begin{array}{c}\text { A } \\
\text { MENUDO } \\
\text { c }\end{array}$ & $\begin{array}{c}\text { SIEMPRE } \\
\text { d }\end{array}$ \\
\hline
\end{tabular}

OBt. Las agresiones son de tipo:

ob01. Físico

ob02. Verbal (insultos, amenazas, etc.)

ob03. Social (exclusión, etc.)

ob04. Psicológico (menosprecio, etc.)

ob05. Sexual

OBr. Las realizó:

ob06. Un/os chico/s

ob07. Una/s chica/s

ob08. Un grupo de chicos y chicas

OBmm. Se producen:

ob09. Durante el trabajo en grupo

ob10. Durante la clase

ob11. En cualquier momento

OBmt. Por qué:

ob12. Por las creencias religiosas, políticas, etc.

ob13. Por la orientación sexual

ob14. Por el aspecto físico

ob15. Por cuestiones académicas

ob16. No lo sé

108. En alguna ocasión, he observado ciberacoso (Agredido a otros mediante el móvil o Internet):
$\mathrm{a} \mathrm{S}$
b $\mathrm{NO}$ 


\begin{tabular}{|c|c|c|c|c|}
\hline & $\begin{array}{c}\text { NUNCA } \\
\text { a }\end{array}$ & $\begin{array}{l}\text { ALGUNAS } \\
\text { VECES } \\
\text { b }\end{array}$ & $\begin{array}{l}\text { A } \\
\text { MENUDO } \\
\text { c }\end{array}$ & $\begin{array}{l}\text { SIEMPRE } \\
\text { d }\end{array}$ \\
\hline \multicolumn{5}{|c|}{ OBCt. He observado ciberacoso (Agredir mediante el móvil o internet): } \\
\hline \multicolumn{5}{|l|}{ obc01. Verbal (insultos, amenazas, etc.) } \\
\hline \multicolumn{5}{|l|}{ obc02. Social (exclusión, etc.) } \\
\hline \multicolumn{5}{|l|}{ obc03. Psicológica (menosprecio, etc.) } \\
\hline obc04. Sexual & & & & \\
\hline \multicolumn{5}{|l|}{ OBCv. Ha sido mediante: } \\
\hline \multicolumn{5}{|l|}{ obc05. Llamadas } \\
\hline \multicolumn{5}{|l|}{ obc06. Mensajería instantánea } \\
\hline \multicolumn{5}{|l|}{ obc07. Mensaje de texto } \\
\hline \multicolumn{5}{|l|}{ obc08. Redes sociales } \\
\hline \multicolumn{5}{|l|}{ obc09. Fotos o vídeos } \\
\hline \multicolumn{5}{|l|}{ OBCx. He observado exclusión en alguien: } \\
\hline \multicolumn{5}{|l|}{ obc10. Del grupo } \\
\hline \multicolumn{5}{|l|}{ obc11. En la toma de decisiones académicas } \\
\hline \multicolumn{5}{|l|}{$\begin{array}{l}\text { obc12. Ocultando o falseando información } \\
\text { relevante de tareas de clase }\end{array}$} \\
\hline \multicolumn{5}{|l|}{$\begin{array}{l}\text { obc13. Ocultando o falseando oras } \\
\text { informaciones (becas, ayudas, etc.) }\end{array}$} \\
\hline \multicolumn{5}{|l|}{ OBCm. Las agresiones se producen por: } \\
\hline \multicolumn{5}{|l|}{ obc14. Las creencias religiosas, políticas, etc. } \\
\hline \multicolumn{5}{|l|}{ obc15. La orientación sexual } \\
\hline \multicolumn{5}{|l|}{ obc16. El aspecto físico } \\
\hline \multicolumn{5}{|l|}{ obc17. Cuestiones académicas } \\
\hline \multicolumn{5}{|l|}{ obc18. No lo sé } \\
\hline \multicolumn{5}{|c|}{ OBCn. Cuando he observado ciberacoso he informado a: } \\
\hline \multicolumn{5}{|l|}{ obc19. Algún compañero/a } \\
\hline \multicolumn{5}{|l|}{ obc20. Algún profesor/a } \\
\hline \multicolumn{5}{|l|}{ obc21. Algún responsable de la institución } \\
\hline \multicolumn{5}{|l|}{ obc22. No he informado } \\
\hline \multicolumn{5}{|c|}{ OBCy. Cuando he observado ciberacoso, he pedido ayuda a: } \\
\hline \multicolumn{5}{|l|}{ obc23. Algún compañero/a } \\
\hline \multicolumn{5}{|l|}{ obc24. Algún profesor/a } \\
\hline \multirow{3}{*}{$\begin{array}{l}\text { obc25. Algún responsable de la institución } \\
\text { obc26. No he pedido ayuda } \\
\text { obc27. No he ofrecido ayuda }\end{array}$} & & & & \\
\hline & & & & \\
\hline & & & & \\
\hline
\end{tabular}

